\title{
A case of ruptured hepatic angiomyolipoma in a young male
}

\author{
Sun Hwa Kim', Tae Wook Kang², Kyunghee Lim', Hyun Sung Joh', Jiseok Kang', and Dong Hyun Sinn \\ Departments of I'Internal Medicine and ${ }^{2}$ Radiology, Samsung Medical Center, Sungkyunkwan University School of Medicine, Seoul, \\ Korea
}

A 31-year-old male visited a local hospital due to sudden-onset severe abdominal pain. Abdominal computed tomography revealed a solid cystic mass with a size of approximately $12 \mathrm{~cm}$ and exhibiting both hemorrhage and fluid collection in the pelvic cavity. Emergency angiography and embolization were performed, and a large hepatic tumor was subsequently surgically resected. The tumor cells stained positive for human melanoma black- 45 and smooth-muscle actin, and the pathologic diagnosis was hepatic angiomyolipoma. This case report also discusses the spontaneous rupture of a hepatic angiomyolipoma. (Clin Mol Hepatol 2017;23:179-183)

Keywords: Angiomyolipoma; Liver; Spontaneous rupture; Human melanoma black-45

\section{INTRODUCTION}

Angiomyolipoma (AML) is an unusual mesenchymal tumor, composed of a varying heterogeneous mixture of three tissue components: blood vessels, smooth muscle, and adipose cells.' AML occurs most commonly in the kidneys, and the liver represents the second most frequent site of involvement. ${ }^{2}$ Tuberous sclerosis may be associated in some cases of $\mathrm{AML}^{3,4}$ but sporadic cases are also frequent.

With the increased use of imaging studies, most cases of AMLs are now discovered incidentally. ${ }^{5}$ However, in renal $A M L$, hemorrhage can be a presenting symptom, and micro-aneurysms are frequently reported. ${ }^{5}$ There is a risk of spontaneous rupture and life threatening hemorrhage in renal $A M L$, especially for lesions $\geq 4 \mathrm{~cm}$ or intralesional aneurysm $>5 \mathrm{~mm}$. $^{4,5}$ These lesions are recommend- ed for consideration of nephrectomy or selective arterial embolization, although surveillance with reimaging is also an option. ${ }^{5}$

In contrast, there is limited information on the risks of rupture and hemorrhage in hepatic AML. Although there are many cases of hepatic AML reported in English literatures, most cases were focused on differential diagnosis of incidentally detected hepatic solid lesions. ${ }^{6}$ As hepatic AML contains an inconstant proportion of fat, smooth muscle and blood vessels, sporadic hepatic AML have a varied appearance, making confident imaging diagnosis difficult in many cases. ${ }^{6}$ Recently, we experienced a case of spontaneous rupture of hepatic AML in a young man, where pre-operative diagnosis was difficult. Herein we report this case with a literature review.

\section{Abbreviations:}

$\mathrm{AML}$, angiomyolipoma; $\mathrm{HMB}$, human melanoma black

\author{
Corresponding author : Dong Hyun Sinn \\ Department of Internal Medicine, Samsung Medical Center, Sungkyunkwan \\ University School of Medicine, 81 Irwon-ro, Gangnam-gu, Seoul 06351, \\ Korea \\ Tel: +82-2-3410-3409, Fax: +82-2-3410-6983 \\ E-mail:dh.sinn@samsung.com \\ http://orcid.org/0000-0002-7126-5554
}

Received : May 3, 2016/ Revised : Jul. 14, 2016/ Accepted : Aug. 8, 2016 


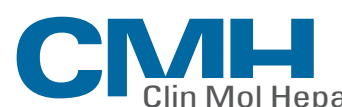

Volume 23 Number 2 June 2017
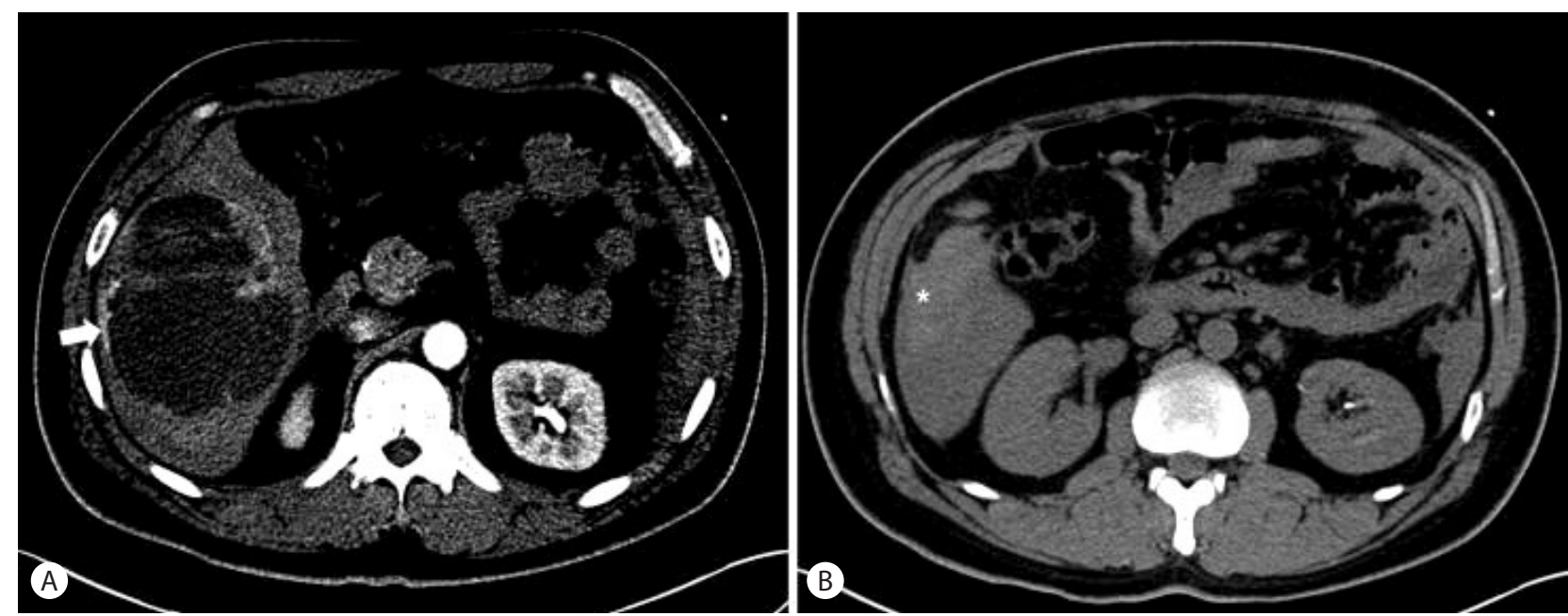

Figure 1. (A) On arterial phase computed tomography (CT) images, a $12 \mathrm{~cm}$ sized mass is noted in the right hepatic lobe. The mass shows a thin peripheral enhancing solid portion (white arrow) with an internal cystic component. (B) On non-enhanced CT scan images, there is a high density (white asterisk) along the perihepatic space representing peritoneal hemorrhage by tumor bleeding.
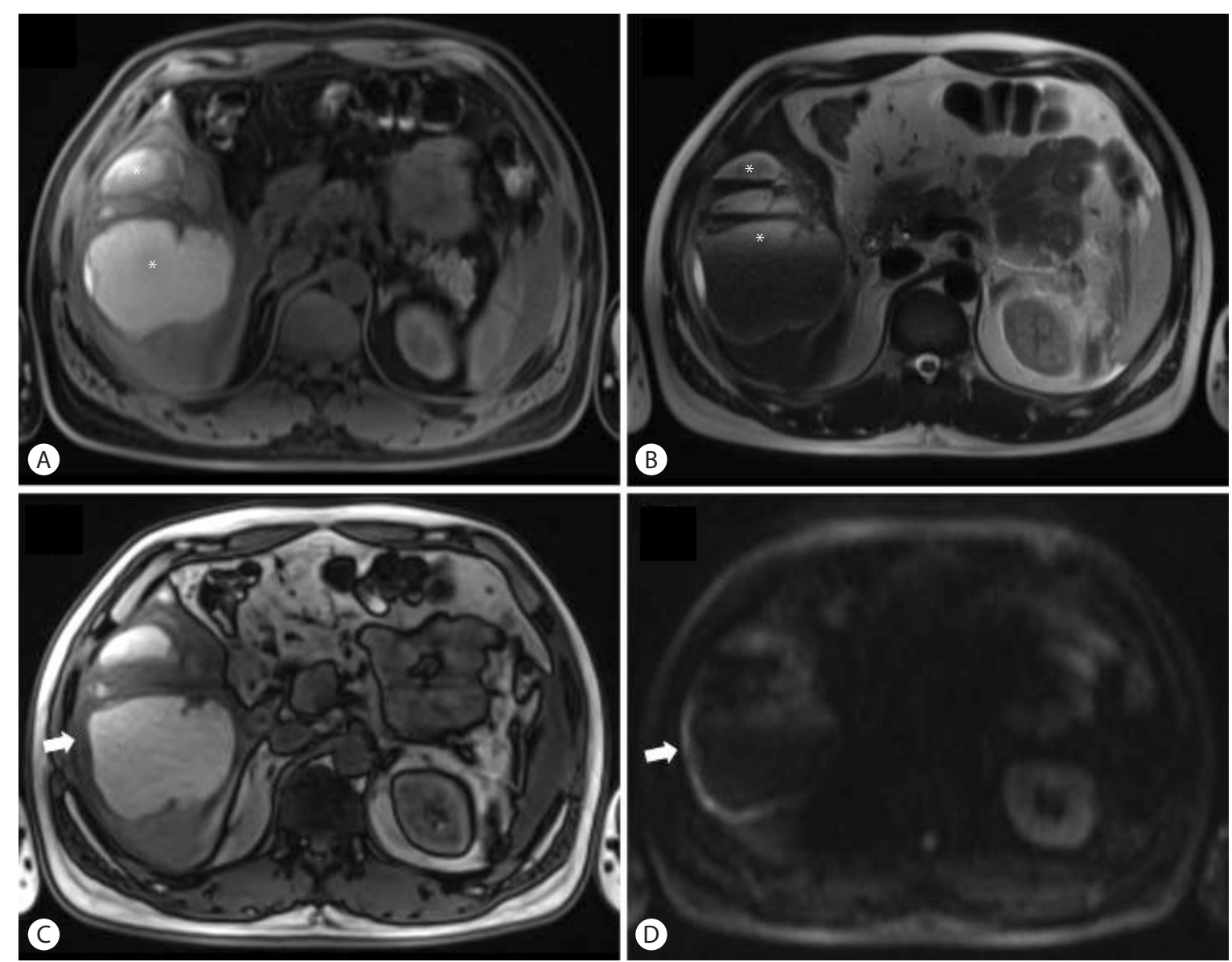

Figure 2. (A, B) On T1 and T2 weighted magnetic resonance (MR) images, the mass has multiloculated cystic lesions with fluid-fluid levels representing internal hemorrhage (white asterisks). (C) On out-of-phase MR images, there is no fat component within the mass (white arrow). (D) On diffusion weighted images with b-value 800, the corresponding peripheral enhancing component shows high signal intensity indicating diffusion restriction (white arrow) 


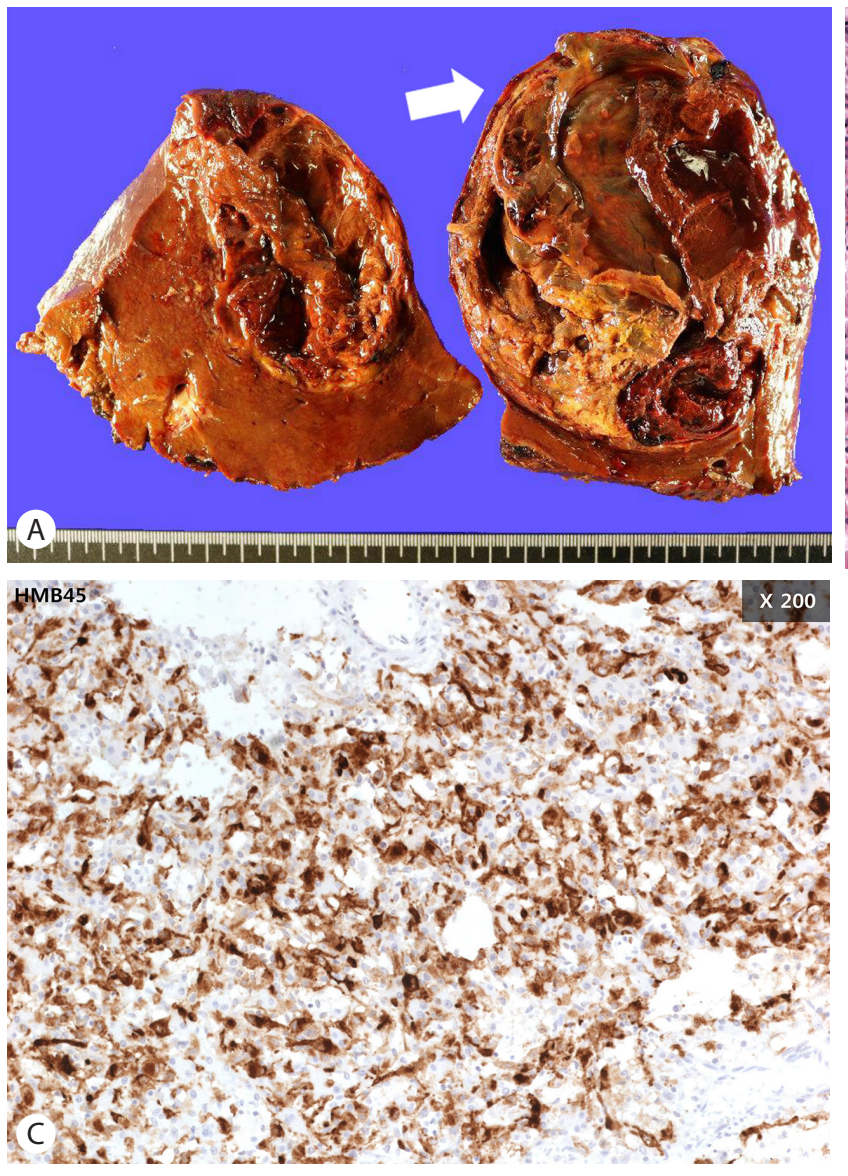

\section{CASE REPORT}

A 31-year-old male was referred to our hospital for a spontaneously ruptured hepatic tumor. Fifteen days earlier, he had presented to the local emergency room due to sudden onset severe abdominal pain localized in the right upper quadrant area. His blood pressure was 130/80 $\mathrm{mmHg}$, heart rate 82 beats/min, body temperature $36.3^{\circ} \mathrm{C}$ and respiratory rate 16 breaths/min. Initial hemoglobin level was $7.1 \mathrm{mg} / \mathrm{dL}$, prothrombin time $76.9 \%$, albumin 4.1 $\mathrm{g} / \mathrm{dL}$, aspartate aminotransferase $20 \mathrm{U} / \mathrm{L}$, and alanine aminotransferase $23 \mathrm{U} / \mathrm{L}$. He complained of severe abdominal discomfort and tenderness on physical exam. The abdominal computed tomography revealed an approximately $12 \mathrm{~cm}$ sized mass in the right hepatic lobe, which showed a thin peripheral enhancing solid portion with an internal cystic component, and accompanying hemorrhage was noted (Fig. 1). Magnetic resonance imaging showed multiloculated cystic lesions with internal hemorrhage. There was no fat component in the enhancing solid portion, and diffusion restriction was also observed (Fig. 2). Emergent angiography with embolization was done, and he was referred to our

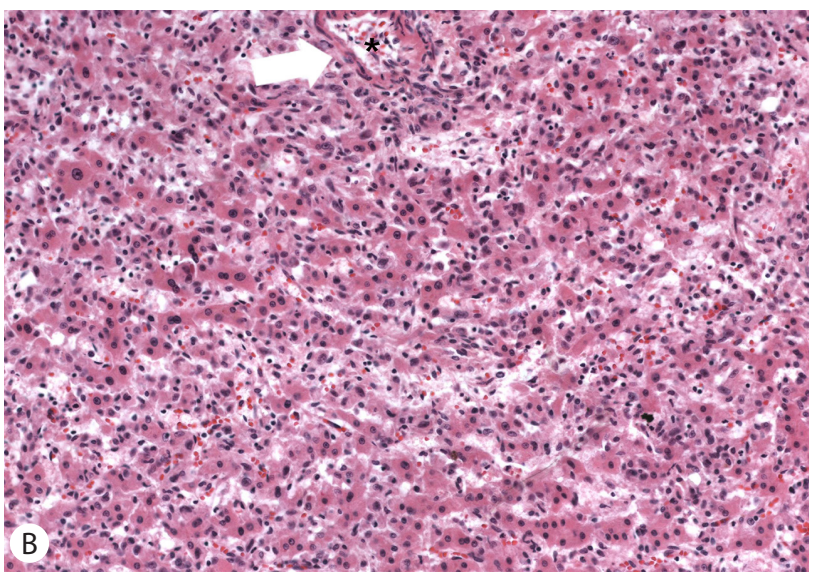

Figure 3. (A) On a cut section, there is a huge multilocular cystic mass (white arrow) measuring $12.5 \times 11 \times 9.9 \mathrm{~cm}$, which is located at S5 and $\mathrm{S} 6$. (B) Spindle or epithelioid smooth muscle cells with eosinophilic cytoplasm and blood vessels (arrow) were seen on pathological findings ( $\mathrm{HE}$, $\times 200$ ). But adipose tissue was rarely seen in this tumor. (C) Result of immunohistochemical staining , HMB-45 (HE, ×200).

hospital. He had no history of diabetes, hypertension or previous surgery. He was not taking any regular medication including anabolic steroid. His blood pressure was $146 / 70 \mathrm{mmHg}$, heart rate 73 beats/min, body temperature $36.8^{\circ} \mathrm{C}$, and respiratory rate 20 breaths/min. Hemoglobin level was $11.3 \mathrm{mg} / \mathrm{dL}$, prothrombin time $85 \%$, albumin $3.8 \mathrm{~g} / \mathrm{dL}$, aspart aminotransferase $14 \mathrm{U} / \mathrm{L}$, and alanine aminotransferase $16 \mathrm{U} / \mathrm{L}$. Hepatitis B surface antigen was negative, anti-hepatitis B surface antibody was positive, and antihepatitis $C$ antibody was negative. Alpha fetoprotein level was 1.3 $\mathrm{ng} / \mathrm{ml}$ and the PIVKA-II test result was $44 \mathrm{mAU} / \mathrm{mL}$. The patient underwent hepatic resection. Pathologically, a huge multilocular cystic mass, measuring $12.5 \times 11.0 \times 9.8$, was noted (Fig. 3A). Microspically extensive necrosis of the tumor (about $60 \%$ ) with a hemorrhage component was observed, without any fat component (Fig. 3B). Immunohistochemical staining showed diffuse positive staining for human melanoma black (HMB)-45 and multifocal positive staining for smooth muscle actin (Fig. 3C). Finally, the tumor was diagnosed as angiomyolipom, of the myomatous type. The patient recovered without complications after surgical resection. 
Table 1. Cases of hepatic AML presenting as a rupture

\begin{tabular}{|c|c|c|c|c|c|}
\hline & Sex & Age & Tumor size $(\mathrm{cm})$ & Symptom & Treatment \\
\hline Huber et al. ${ }^{16}(1996)$ & $\mathrm{F}$ & 22 & 8 & Hemorrhagic shock & Emergent resection \\
\hline Guidi et al." (1997) & M & 74 & 10 & Upper abdominal pain & Emergent resection \\
\hline Tsui et al. ${ }^{8}(1999)$ & M & 56 & 6.5 & N/A & Emergent resection \\
\hline Zhou et al. ${ }^{15}$ (2008) & N/A & N/A & 5 & Hemorrhagic shock & Emergent laparotomy for hemostasis \\
\hline Occhionorelli et al. ${ }^{12}(2013)$ & F & 25 & 9 & Upper abdominal pain & $\begin{array}{l}\text { Elective resection after emergent laparotomy } \\
\text { for hemostasis }\end{array}$ \\
\hline Tajima et al. ${ }^{13}$ (2014) & M & 38 & 10.5 & Upper abdominal pain & $\begin{array}{l}\text { Elective resection after transcatheter arterial } \\
\text { embolization }\end{array}$ \\
\hline Kai et al. ${ }^{14}(2015)$ & F & 77 & 2.5 & Hemorrhagic shock & $\begin{array}{l}\text { Elective resection after transcatheter arterial } \\
\text { embolization }\end{array}$ \\
\hline This case & M & 31 & 12.5 & Upper abdominal pain & $\begin{array}{l}\text { Elective resection after transcatheter arterial } \\
\text { embolization }\end{array}$ \\
\hline
\end{tabular}

AML, angiomyolipoma; N/A, not applicable.

\section{DISCUSSION}

Hepatic AML is a tumor capable of dual myomatous and lipomatous differentiation and melanogenesis, with HMB-45 positive myoid cells as the defining criterion. ${ }^{7.8}$ Because of its protean morphologic appearance, various variant patterns have been recognized such as mixed, lipomatous, myomatous, and angiomatous types, including the epitheloid type with no or a minimal amount of fat. ${ }^{9}$ Hence, a correct preoperative diagnosis of hepatic AML is demonstrated in only $20-52 \%$ of cases, especially in lipid poor AML. ${ }^{9}$

The distinction of fat-deficient AML from other tumors, especially HCC, is very challenging. Jeon et al. suggested that the presence of early draining veins connected to prominent tumoral vessels and the absence of tumor capsules may help with the differentiation of fat-deficient AML in non-cirrhotic liver. ${ }^{10} \mathrm{Kim}$ et al. suggested homogeneous hypointensity in the hepatobiliary phase in gadoxetic acid-enhanced magnetic resonance imaging. ${ }^{9}$ However, these findings were not observed in this case.

In differential diagnosis, demographic or clinical findings can be helpful. As malignant tumors such as hepatocellular carcinoma mostly develops in patients with risk factors (e.g., hepatitis B, hepatitis $C$ or cirrhosis), identifying risk factors for hepatocellular carcinoma development is very important for differential diagnosis. ${ }^{7}$ Age, sex and clinical behaviors also help with differential diagnosis. Hepatic adenoma can be suspected for women taking oral contraceptive therapy. Hemorrhage has been reported in 11$29 \%$ of hepatocellular adenoma cases, with nearly all instances of spontaneous rupture occurring in lesions $\geq 5 \mathrm{~cm}^{7}$. In cases of hepatic AML, ruptures are usually observed in middle aged women. ${ }^{8}$ Spontaneous rupture and hemorrhage is uncommon, and to our best knowledge, there are only seven cases of hepatic AML presenting as a rupture (Table 1). ${ }^{8,11-16}$ The size of the tumors are usually large, and there are no gender or age preferences in the reported cases. In this case, the patient was a young man without risk factors for hepatocellular carcinoma and presented with a spontaneous rupture, fat-deficient case. Differential diagnosis was very challenging. The characteristic findings of hepatic AML are the presence of both fat and prominent vascularity in the same lesion. Conversely, less fatty and hypervascular hepatic mass accompanying hemorrhage radiologically suggested hepatocellular carcinoma or hepatic adenoma. As he had no risk factors for hepatocellular carcinoma, the first differential diagnosis was hepatic adenoma in this case. The correct diagnosis could only be confirmed after resection of the tumor by immunohistochemical staining. In summary, we present a case of ruptured hepatic AML in a young male patient. This variant of hepatic AML should be included as a differential diagnosis of hepatic tumor presenting as a rupture.

\section{Conflicts of Interest}

The authors have no conflicts to disclose.

\section{REFERENCES}

1. Xu AM, Zhang SH, Zheng JM, Zheng WQ, Wu MC. Pathological and molecular analysis of sporadic hepatic angiomyolipoma. Hum Pathol 2006;37:735-741. 
2. Petrolla AA, Xin W. Hepatic angiomyolipoma. Arch Pathol Lab Med 2008;132:1679-1682.

3. Lenci I, Angelico M, Tisone G, Orlacchio A, Palmieri G, Pinci M, et al. Massive hepatic angiomyolipoma in a young woman with tuberous sclerosis complex: significant clinical improvement during tamoxifen treatment. J Hepatol 2008;48:1026-1029.

4. Jinzaki M, Silverman SG, Akita H, Nagashima Y, Mikami S, Oya M. Renal angiomyolipoma: a radiological classification and update on recent developments in diagnosis and management. Abdom Imaging 2014;39:588-604.

5. Flum AS, Hamoui N, Said MA, Yang XJ, Casalino DD, McGuire BB, et al. Update on the Diagnosis and Management of Renal Angiomyolipoma. J Urol 2015;195:836-846.

6. Low SC, Peh WC, Muttarak M, Cheung HS, Ng IO. Imaging features of hepatic angiomyolipomas. J Med Imaging Radiat Oncol 2008;52:118-123.

7. Marrero JA, Ahn J, Rajender Reddy K. ACG clinical guideline: the diagnosis and management of focal liver lesions. Am J Gastroenterol 2014;109:1328-1347.

8. Tsui WM, Colombari R, Portmann BC, Bonetti F, Thung SN, Ferrell LD, et al. Hepatic angiomyolipoma: a clinicopathologic study of 30 cases and delineation of unusual morphologic variants. Am J Surg Pathol 1999;23:34-48.

9. Kim R, Lee JM, Joo I, Lee DH, Woo S, Han JK, et al. Differentiation of lipid poor angiomyolipoma from hepatocellular carcinoma on gadoxetic acid-enhanced liver MR imaging. Abdom Imaging 2015;40:531-
541.

10. Jeon TY, Kim SH, Lim HK, Lee WJ. Assessment of triple-phase CT findings for the differentiation of fat-deficient hepatic angiomyolipoma from hepatocellular carcinoma in non-cirrhotic liver. Eur J Radiol 2010;73:601-606.

11. Guidi G, Catalano O, Rotondo A. Spontaneous rupture of a hepatic angiomyolipoma: CT findings and literature review. Eur Radiol 1997;7:335-337.

12. Occhionorelli S, Dellachiesa L, Stano R, Cappellari L, Tartarini D, Severi $S$, et al. Spontaneous rupture of a hepatic epithelioid angiomyolipoma: damage control surgery. A case report. G Chir 2013;34:320322.

13. Tajima S, Suzuki A, Suzumura K. Ruptured hepatic epithelioid angiomyolipoma: a case report and literature review. Case Rep Oncol 2014;7:369-375

14. Kai K, Miyosh A, Aishima S, Wakiyama K, Nakashita S, Iwane S, et al. Granulomatous reaction in hepatic inflammatory angiomyolipoma after chemoembolization and spontaneous rupture. World J Gastroenterol 2015;21:9675-9682.

15. Zhou YM, Li B, Xu F, Wang B, Li DQ, Zhang XF, et al. Clinical features of hepatic angiomyolipoma. Hepatobiliary Pancreat Dis Int 2008;7:284-287.

16. Huber C, Treutner KH, Steinau G, Schumpelick V. Ruptured hepatic angiolipoma in tuberous sclerosis complex. Langenbecks Arch Chir 1996;381:7-9. 